\title{
Physics of Low Voltage Scanning Electron Microscopy
}

\author{
Raynald Gauvin
}

Department of Mining, Metals and Materials Engineering, McGill University, Montréal, Québec, Canada, H3A 2B2

With the advent of the Field Emission Scanning Electron Microscopes (FE-SEM), images can be acquired routinely with incident electron energies as low as $500 \mathrm{eV}$. Recently, images acquired in a FE-SEM with incident electron energy of $50 \mathrm{eV}$ have been reported ${ }^{1}$. In this context, it is critical to model electron scattering in solids at such low electron energy. Despite the success of Monte Carlo simulations based on the Continuous Slowing Down Approximation ${ }^{2}$, it is clear that a different model should be used to compute energy loss for electron energy below $1 \mathrm{keV}$.

To model electron scattering below $1 \mathrm{keV}$, the probability of inelastic collision must be computed at each collision and if an inelastic collision occurs, energy loss must be computed using an appropriate distribution obtained from an appropriate physical model. The dielectric theory allows computing such an energy loss distribution function if the complex dielectric constant of the simulated material is known. This complex dielectric constant can be determined for a specific material from an Electron Energy Loss Spectroscopy (EELS) experiment ${ }^{3}$.

In order to validate a Monte Carlo program that computes discrete energy loss at each inelastic collision, comparison with measured signals from this materials must be performed. Figure [1] shows all the measured backscattering coefficients reported so far for gold ${ }^{4}$. Below $2 \mathrm{keV}$, the scattering of the experimental measurements is about $50 \%$. It is clear that the validation of Monte Carlo programs for energy smaller than $2 \mathrm{keV}$ is impossible with such a lack of good experimental data. As seen in figure [2], the situation is even worse for the measured secondary electron yields of silicon ${ }^{4}$. The scatter of experimental data below $2 \mathrm{keV}$ is about $200 \%$. Clearly, very accurate measurements of fundamental parameters like backscattering coefficients and secondary electron yields are needed in order to validate Monte Carlo models of electron scattering at low electron energy.

\section{References}

1. D. C. Joy and C. S. Joy (1996), Proceedings of Microscopy \& Microanalysis, pp. 144 -145 .

2. P. Hovington, D. Drouin and R. Gauvin (1997), Scanning, Vol.19, pp. 1-14.

3. D.C. Joy, S. Luo, R. Gauvin, P. Hovington and N. Evans (1996), Scanning Microscopy, Vol. 16, pp. 209-220.

4. D.C. Joy (2001), Microscopy \& Microanalysis, Vol. 7, pp. 159 - 167. 
Au

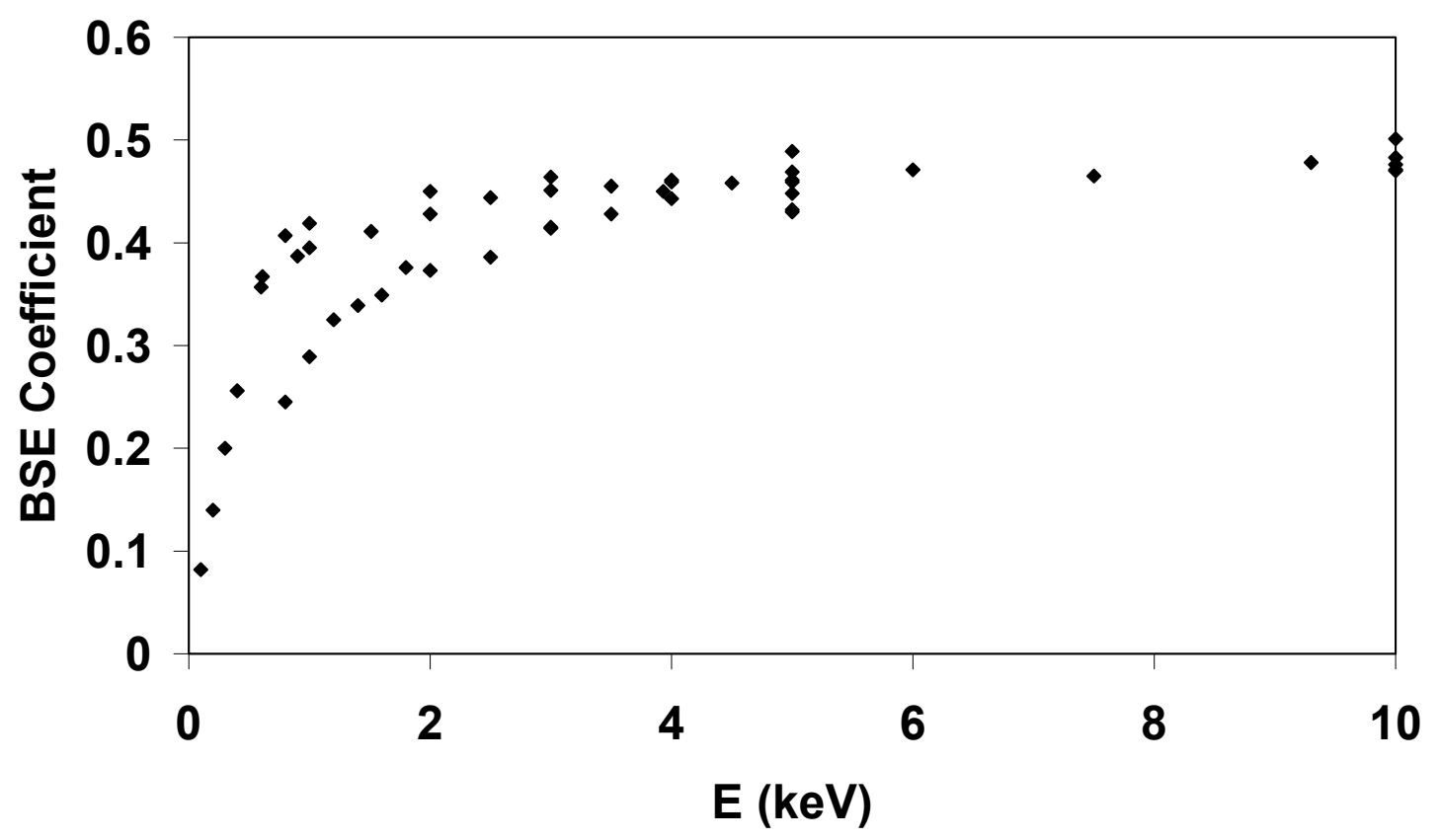

Figure 1 Measured Backscattered Electron coefficient of Gold as a function of $E_{0}$.

\section{Silicon}

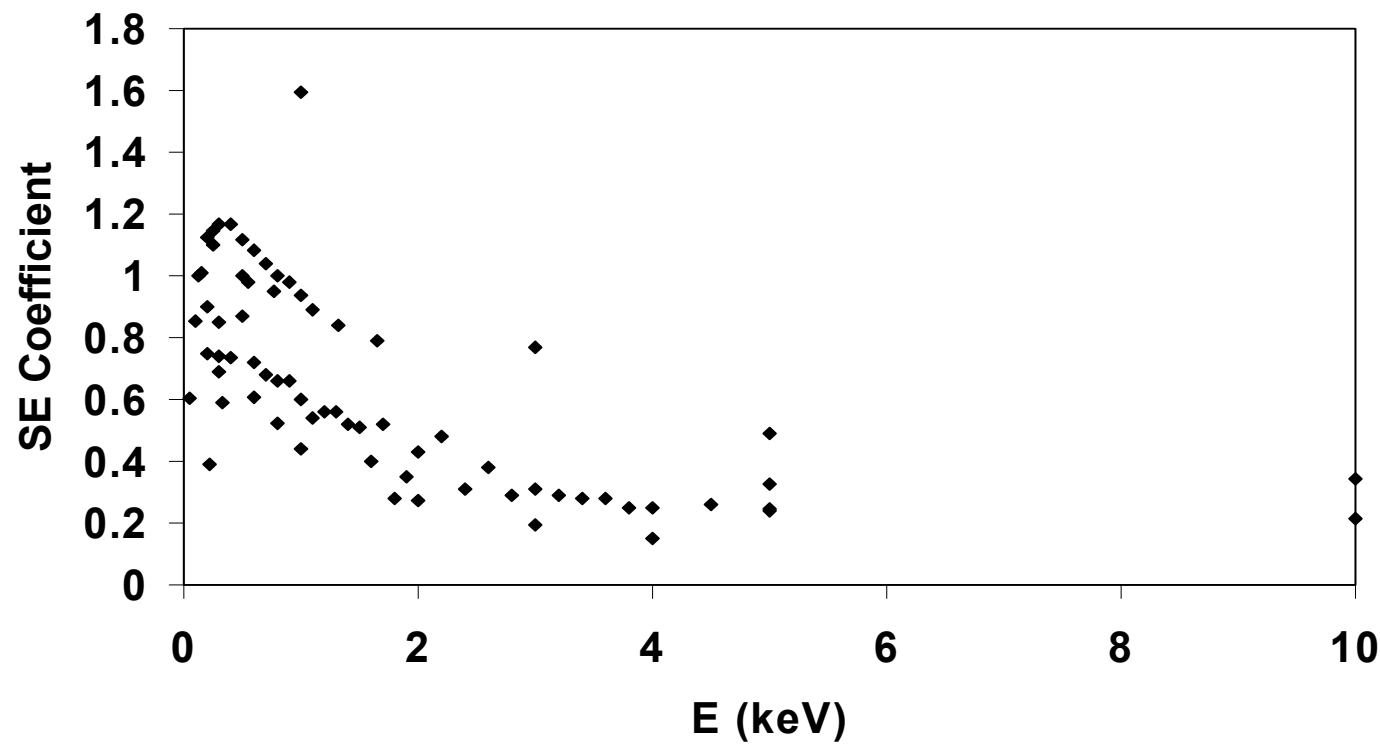

Figure 2 Measured Secondary Electron coefficient of $S i$ as a function of $E_{0}$. 
https://doi.org/10.1017/S1431927602101905 Published online by Cambridge University Press 